[Radiocarbon, Vol. 20, No. 1, 1978, P. 58-61]

\title{
NUCLEAR SCIENCE AND TECHNOLOGY FACILITY RADIOCARBON DATES I
}

\author{
G C THOMAS, JR and F C THOMAS
}

Radiocarbon Laboratory, Nuclear Science and Technology Facility, State University of New York at Buffalo, Buffalo, New York 14214

The Radiocarbon Laboratory began operation in mid-1972, using benzene synthesis. The equipment for the laboratory was obtained under a National Science Foundation Grant*. Basic equipment consists of a benzene synthesizer (Alford Instrument Co) and a liquid scintillation counter (Picker-Nuclear 220). The equipment is essentially identical with that of Noakes and Brandau (1971). Counting efficiency and background rates are also similar; ca $70 \%$ efficiency with a 4 to $5 \mathrm{cpm}$ background for $5 \mathrm{ml}$ benzene samples. Benzene synthesis is standard with sample combustion in pure oxygen under reduced pressure. The original synthesis system has been modified to permit simultaneous processing of more than one sample.

Dates are based on 0.95 of the activity of National Bureau of Standards oxalic acid and the Libby half-life for ${ }^{14} \mathrm{C}$. Counting techniques and data analysis procedures are similar to those described by Polach (1969) with the exception that Tamers' (1965) bottles are used. $\delta^{13} \mathrm{C}$ values are currently based on data reported in Radiocarbon. Dates for various types of samples have been averaged and the standard deviation calculated. Recent acquisition of a mass spectrometer and a qualified operator will permit use of measured $\delta^{13} \mathrm{C}$ values.

Samples dated thus far have been primarily wood or charcoal, although some peat samples have been dated. Pretreatment of wood and charcoal is a standard acid-alkali procedure, using $2 \% \mathrm{HCl}$ and $2 \% \mathrm{NaOH}$ at elevated temperature. Peat is initially suspended in water, sieved, and then subjected to treatment with acid and alkali at ambient or slightly elevated temperatures. Contaminating materials such as rootlets and pebbles are mechanically removed.

As a result of limited personnel, the laboratory has functioned well below capacity since completion of the equipment installation, and preliminary testing in early 1973 . Dating has been done by the authors with the assistance of graduate and undergraduate students. Dates obtained on previously dated wood and charcoal specimens are shown in Table 1, and demonstrate that the dates obtained by the laboratory are in good agreement with the original dates.

* National Science Foundation Grant SG-28297, “Specialized Equipment for Radiocarbon Dating Facility." 
TABLE 1

Interlaboratory Sample Dates*

\begin{tabular}{lclll}
\hline $\begin{array}{c}\text { NSTF } \\
\text { sample no. }\end{array}$ & \multicolumn{1}{c}{$\begin{array}{c}\text { NSTF } \\
\text { date }\end{array}$} & $\begin{array}{c}\text { Original } \\
\text { sample no. }\end{array}$ & $\begin{array}{c}\text { Original } \\
\text { dating lab }\end{array}$ & $\begin{array}{c}\text { Original } \\
\text { date }\end{array}$ \\
\hline NSTF-2 & $1515 \pm 290 * *$ & GX-2072 & Geochron & $1655 \pm 80$ \\
NSTF-3 & $1759 \pm 176$ & GX-2142 & Geochion & $1695 \pm 90$ \\
NSTF-23 & $10,188 \pm 396$ & SI-1329 & Smithsonian & $9835 \pm 90$ \\
NSTF-53 & $6846 \pm 187$ & BGS-120 & Brock Univ & $6800 \pm 120$ \\
\hline
\end{tabular}

* Radiocarbon years BP

** Minimum sample size

The sample site descriptions provided for early samples were less than complete. As a result, the archaeologist submitting the sample and his affiliation are noted in the following list.

\section{ACKNOWLEDGMENTS}

The authors gratefully acknowledge the invaluable technical advice and assistance provided by H Polach, J Noakes, and R Stuckenrath. The efforts of J Y Yang in the initial setup and testing of the benzene synthesizer were of considerable value to the establishment of the laboratory. Finally, the assistance of the following graduate and undergraduate students in dating samples is gratefully acknowledged: BSA Herbert, G Botham, MS Thomas, and L Boylan.

\section{ARCHAEOLOGIC SAMPLES}

\section{A. Mexico}

\section{NSTF-5. Arrinitas-la}

$$
\begin{array}{lr}
\delta^{14} \mathbf{C}=-\mathbf{8 1 . 5} \% & \mathbf{6 8 5} \pm \mathbf{8 5} \\
\Delta \quad=-81.7 \% & \delta^{13} C=-25.1 \pm 1.29 \%
\end{array}
$$

Charcoal from Arrinitas, Mound A, Sq A, Level 7, Lot $7\left(22^{\circ} 29^{\prime} \mathrm{N}\right.$, $105^{\circ} 39^{\prime}$ W). Subm by S Scott, State Univ New York at Buffalo.

$\begin{array}{llr}\text { NSTF-9. Arrinitas-lg } & \delta^{14} \mathrm{C}=-\mathbf{1 2 8 . 9} \% \circ & 1108 \pm 62\end{array}$

$$
\Delta=-\mathbf{1 2 8 . 8} \% \quad \delta^{13} \mathrm{C}=-25.1 \pm 1.29 \%
$$

Charcoal from Arrinitas, Mound A, Sq A, Level 8, 3.5 from NW stake in SE quad $\left(22^{\circ} 29^{\prime} \mathrm{N}, 105^{\circ} 39^{\prime} \mathrm{W}\right)$. Subm by S Scott.

$$
\begin{array}{rlr}
\text { NSTF-7. Tecicallo-la } & \boldsymbol{\delta}^{14} \mathbf{C}=-\mathbf{1 1 0 . 9 5} \% & \mathbf{9 4 6} \pm \mathbf{6 0} \\
\Delta \quad & =-111.13 \% \circ & \boldsymbol{\delta}^{13} C=-24.9 \pm 1.78 \% \mathrm{co}
\end{array}
$$

Charcoal from Tecicallo, Mound Feature A, Level $5\left(22^{\circ} 28^{\prime} \mathrm{N}\right.$, $\left.105^{\circ} 39^{\prime} \mathrm{W}\right)$. Subm by S Scott.

\section{NSTF-6. Tecicallo}

Charcoal from Tecicallo, Mound A, Feature A, Level $4\left(22^{\circ} 28^{\prime} \mathrm{N}\right.$, $\left.105^{\circ} 39^{\prime} \mathrm{W}\right)$. Subm by S Scott. 


$$
\text { B. New York }
$$

NSTF-11R. Witch's Walk $\delta^{14} \mathrm{C}=-\mathbf{1 8 3 . 6 0} \%$

$$
\Delta=-183.77 \% \text { o } \delta^{13} \mathrm{C}=-24.9 \pm 1.78 \%
$$

Charcoal from Witch's Walk Iroquois site, Sq $25 \mathrm{~W}, 15 \mathrm{E}$, Feature 1 $\left(42^{\circ} 6^{\prime} 18^{\prime \prime} \mathrm{N}, 78^{\circ} 50^{\prime} 25^{\prime \prime} \mathrm{W}\right)$. Subm by J Schock, State Univ New York at Buffalo.

$$
\begin{aligned}
& \text { NSTF-13R. Lawrence } \delta^{1 t} \mathrm{C}=-\mathbf{1 2 7 . 4 2} \% \text { 1097 } \pm 65 \\
& \Delta=-\mathbf{1 2 7 . 6 0} \% \quad \delta^{13} \mathrm{C}=-24.9 \pm 1.78 \% \text { o }
\end{aligned}
$$

Charcoal from Lawrence Iroquois site, Feature $8\left(42^{\circ} 14^{\prime} 32^{\prime \prime} \mathrm{N}\right.$, $\left.79^{\circ} 08^{\prime} 13^{\prime \prime} \mathrm{W}\right)$. Assoc with pottery and triangular points. Subm by J Schock.

$$
\begin{aligned}
& \text { NSTF-14. Falcone } \quad \delta^{14} \mathrm{C}=-\mathbf{7 2 . 1 9} \% \\
& \Delta=-\mathbf{7 2 . 3 7} \% \quad \delta^{13} C=-24.9 \pm 1.78 \%
\end{aligned}
$$

Charcoal from Falcone Iroquois site, Feature $8\left(42^{\circ} 14^{\prime} 54^{\prime \prime} \mathrm{N}\right.$, $\left.79^{\circ} 15^{\prime} 42^{\prime \prime} \mathrm{W}\right)$. Round pit with flint content. Assoc with flint and pottery. Subm by J Schock.

$$
\begin{aligned}
& \text { NSTF-15. Falcone } \quad \delta^{14} \mathrm{C}=-\mathbf{8 8 . 5 3} / / c \quad \mathbf{7 4 6} \pm \mathbf{5 7} \\
& \Delta=-88.71 \% 0 \quad \delta^{13} C=-24.9 \pm 1.78 \% c
\end{aligned}
$$

Charcoal from Falcone Iroquois site, Feature $7\left(42^{\circ} 14^{\prime} 54^{\prime \prime} \mathrm{N}\right.$, $\left.79^{\circ} 15^{\prime} 42^{\prime \prime} \mathrm{W}\right)$. Top level from pit with high sherd and flint content. Reddened soil above top level. Assoc with pottery and flint. Sub by J Schock.

$$
\begin{aligned}
& \text { NSTF-18. Ellington } \quad \delta^{14} \mathrm{C}=-\mathbf{7 4 . 3 1} \% / c \quad 622 \pm \mathbf{1 0 7} \\
& \Delta=-74.49 \% \quad \delta^{13} C=-24.9 \pm 1.78 \%
\end{aligned}
$$

Charcoal from Ellington Iroquois site $\left(42^{\circ} 8^{\prime} 16^{\prime \prime} \mathrm{N}, 79^{\circ} 6^{\prime} 16^{\prime \prime} \mathrm{W}\right)$. From near base of depression with much midden material. Subm by J Schock.

$$
\begin{aligned}
& \text { NSTF-19C. Rhinehart } \delta^{1+} \mathrm{C}=-\mathbf{1 0 9 . 8 7} \% \% \\
& \Delta=-\mathbf{1 1 0 . 0 5} \% \quad \boldsymbol{\delta}^{13} \mathrm{C}=-24.9 \pm 1.78 \%
\end{aligned}
$$

Charcoal, NSTF 16, 17, and 19 combined. Samples from Rhinehart Iroquois site $\left(42^{\circ} 14^{\prime} 21^{\prime \prime} \mathrm{N}, 79^{\circ} 07^{\prime} 37^{\prime \prime} \mathrm{W}\right)$. From base of earthring Trench B. Assoc with pottery and flint. Sulsm by J Schock.

\section{Pennsyluania}

$$
\text { NSTF-47. Swainville } \begin{array}{rr}
\delta^{14} \mathrm{C} & =\mathbf{- 2 2 3 . 4 2} \% \\
\Delta \quad & =-\mathbf{2 2 3 . 2 7} \%
\end{array} \quad \begin{array}{r}
\mathbf{2 0 3 0} \pm \mathbf{9 8} \\
\delta^{13} C=-25.1 \pm 1.29 \%
\end{array}
$$

Wood from Swainville site $\left(42^{\circ} 4^{\prime} \mathrm{N}, 80^{\circ} 11^{\prime} \mathrm{W}\right)$. Pit in sandy gravel. Sample from lens overlain with small sec of humus and balance sand. Sample depth, $0.46 \mathrm{~m}$. Slightly damp soil (seasonal fluctuation) $\mathrm{pH} 5.4$. Subm by W Englebrecht, State Univ New York at Buffalo. 
NSTF-48. Swainville $\quad \delta^{14} \mathrm{C}=-\mathbf{2 0 0 . 1 7} \%$

$1793 \pm 90$

$\Delta=-\mathbf{2 0 0 . 0 1} \% \quad \delta^{13} \mathrm{C}=-25.1 \pm 1.29 \%$

Wood from Swainville site (NSTF-47). Sample depth, 0.64 to $0.88 \mathrm{~m}$. Subm by W Englebrecht.

\section{GEOLOGIC SAMPLES}

A. New York

NSTF-24. Huff Island

Fibrous peat from 90 to $100 \mathrm{~cm}$ depth. Subm by L C Marsh, State Univ New York at Oswego.
NSTF-25. Huff Island $\delta^{14} \mathrm{C}=-\mathbf{4 6 1 . 6 4} \%$
$\Delta=-\mathbf{4 5 8 . 8 4} \% \quad \delta^{13} \mathrm{C}=-27.6 \pm 2.24 \%$

Peat with wood from 220 to $230 \mathrm{~cm}$ depth. Subm by L C Marsh.
NSTF-27. Huff Island $\delta^{14} \mathbf{C}=-\mathbf{5 0 8 . 9} \%$
$5672 \pm 337$
$\Delta=-\mathbf{5 0 6 . 3 5} \% \quad \delta^{13} \mathrm{C}=-27.6 \pm 2.27 \%$

Peat with some marl and shells from 420 to $430 \mathrm{~cm}$ depth. Subm by L C Marsh.

NSTF-29. Jordan Station $\quad \delta^{14} \mathrm{C}=-\mathbf{1 8 2 . 7 6} \%$ $\Delta=-\mathbf{1 7 8 . 5 1} \% \quad \delta^{13} \mathrm{C}=-27.6 \pm 2.24 \%$

Peat from 240 to $250 \mathrm{~cm}$ depth. Subm by L C Marsh.
NSTF-32. Buck Pond $\delta^{1+} \mathrm{C}=-\mathbf{1 4 9 . 1 1} \%$
$1255 \pm 58$
$\Delta=-\mathbf{1 4 4 . 6 9} \% \quad \delta^{13} \mathrm{C}=-27.6 \pm 2.27 \%$

Peat from 245 to $255 \mathrm{~cm}$ depth. Subm by L C Marsh.
NSTF-38. Port Bay
$\begin{aligned} \delta^{14} \mathbf{C} & =-630.6 \% \\ \Delta & =-628.7 \%\end{aligned}$
$7959 \pm 110$
$\delta^{1.3} \mathrm{C}=-27.6 \pm 2.27 \%$

Fibrous peat from 90 to $100 \mathrm{~cm}$ depth. Date appears too old. Subm by L C Marsh.

\section{REFERENCES}

Noakes, J E and Brandau, B L, 1971, University of Georgia radiocarbon dates I: Radiocarbon, v 13, p 468-474.

Polach, H A, 1969, Optimization of liquid scintillation radiocarbon age determinations and reporting of ages: Atomic Energy in Australia, v 12, no. 3, p 21-28.

Tamers, M A, 1965, Routine carbon 14 dating using liquid scintillation technique: 6th internatl conf on radiocarbon and tritium dating, Proc, Pullman, Washington, p 53-67. 